\title{
Control-based Continuation of Unstable Periodic Orbits
}

\author{
Jan Sieber*
}

\author{
Bernd Krauskopf $^{\dagger}$ \\ David Wagg ${ }^{\ddagger}$ \\ Alicia Gonzalez-Buelga!
}

Simon Neild ${ }^{\S}$

\begin{abstract}
We present an experimental procedure to track periodic orbits through a fold (saddle-node) bifurcation, and demonstrate it with a parametrically excited pendulum experiment where the control parameter is the amplitude of the excitation. Specifically, we track the initially stable periodone rotation of the pendulum through its fold bifurcation and along the unstable branch. The fold bifurcation itself corresponds to the minimal amplitude that supports sustained rotation. Our scheme is based on a modification of timedelayed feedback in a continuation setting, and we show for an idealized model that it converges with the same efficiency as classical proportional-plus-derivative control.
\end{abstract}

\section{Contents}

1 Introduction

2 Feedback controlled experiment as a nonlinear fixed point problem

3 Applying time-delayed feedback control

4 Experimental implementation

5 Experimental results

6 Conclusion

\section{Introduction}

If a dynamical system is given in the form of a system of ordinary differential equations (ODEs) then the continuation of time-periodic motions and their bifurcations can be performed with existing numerical continuation software $[6,14,5]$. The availability of these numerical tools has been a breakthrough in the analysis of low-dimensional systems of ODEs. Similar methods have been developed also for high- or infinite-dimensional systems with 'essentially lowdimensional dynamics' such as delay differential equations,

\footnotetext{
*Dept. of Mathematics, University of Portsmouth, PO1 3HF, Portsmouth, United Kingdom,

${ }^{\dagger}$ Dept. of Engineering Mathematics, University of Bristol, Bristol, BS8 1TR, United Kingdom

${ }^{\ddagger}$ Dept. of Mechanical Engineering, University of Bristol, Bristol, BS8 1TR, United Kingdom

${ }^{\S}$ Dept. of Mechanical Engineering, University of Bristol, Bristol, BS8 1TR, United Kingdom

`Dept. of Civil Engineering, Universitat Politècnica de Catalunya, 08034 Barcelona, Spain
}

dissipative partial differential equations, or Monte-Carlo simulations $[7,15,13]$. One reason behind the success of these methods is that they make phenomena visible that are notoriously difficult to find in simulations and experiments, for example, due to their dynamical instability or extreme sensitivity with respect to changes in system parameters.

In [20] we have proposed a combination of Newton iterations and standard feedback control that can be embedded into a continuation to perform some bifurcation analysis tasks directly in experiments. Two features of the scheme make it especially suitable for experiments:

1. The accuracy of the results does not rely on the accuracy of a model (and thus, it does not require system identification, which is an inverse problem) but only on measurement tolerances and the condition number of the Jacobian in the Newton iteration, which is estimated as part of the procedure.

2. It is not necessary to set initial conditions for the state of the dynamical system.

2 The main requirement for the applicability of the scheme is the presence of a stabilizing feedback loop. One class of experiments where tunable feedback loops are naturally present are so-called hybrid tests in civil and mechanical engineering, which bidirectionally couple a mechanical experiment with a computer simulation $[4,10]$.

In [19] we have demonstrated the practical feasibility of this control-based continuation in a simple mechanical prototype experiment - the parametrically excited pendulum. Independently, the experiments in [1] applied this approach to trace out unstable oscillations of a nonlinear energy harvester. In this paper we present the technical details of the procedure and the complete results of the pendulum experiment in [19]. Furthermore, we analyze the convergence of the scheme over a large parameter range for an idealized model.

\section{Feedback controlled experiment as a nonlinear fixed point problem}

The key idea behind our method is that the introduction of a stabilizing feedback loop into an experiment (representing a smooth nonlinear dynamical system) turns this experiment into a nonlinear fixed point problem. The solutions of this fixed point problem are the natural trajectories of the uncontrolled experiment. In this section we discuss this approach with the simple model of the parametrically excited pendulum; the practical implementation is discussed in Section 4. 
The dynamics of a parametrically excited pendulum is given in good approximation by the ODE

$$
m l^{2} \ddot{\theta}(t)+b \dot{\theta}(t)+m l[g+\ddot{y}(t)] \sin \theta(t)=0 .
$$

In (1), $\theta$ is the angle of the pendulum, $y(t)$ is the excitation amplitude of the pivot (in meters), $b$ is the viscous friction coefficient, $m$ is the effective mass of the pendulum, $l$ is the effective length of the pendulum, and $g$ is the acceleration due to gravity. A typical choice for the excitation would be a harmonic of amplitude $p$ and frequency $\omega$, as given by $y(t)=p \sin (\omega t)$. The pendulum with harmonic parametric excitation is a well-studied system; its bifurcation diagram in the $(\omega, p)$-plane can be found, for example, in [21]. We focus here on a particular type of dynamics, namely period-one rotations of the pendulum. They are given by functions that satisfy $\theta(t)=\theta(t+2 \pi / \omega)-2 \pi$ if they rotate in the positive direction (the long-time average of $\dot{\theta}$ is positive). Period-one rotations are periodic orbits of (1) in rotating coordinates. That is, introducing $\phi=\theta-\omega t$ and assuming harmonic excitation $y(t)$, the angle variable $\phi$ satisfies the differential equation

$$
m l^{2} \ddot{\phi}+b \dot{\phi}+b \omega+m l\left[g-\omega^{2} p \sin (\omega t)\right] \sin (\phi+\omega t)=0 .
$$

A solution $\phi(t)$ is a periodic orbit of (2) if it satisfies $\phi(t)=$ $\phi(t+2 \pi / \omega)$ for all times $t$.

In our experimental implementation for practical reasons the control input $u(t)$ is super-imposed onto the harmonic excitation $y(t)$; see Section 4 for details. However, in our analysis we assume for simplicity that we can apply control in its idealized form as a torque at the pivot such that $u$ enters on the right-hand-side of (2). We also assume that $\theta(t)$ and $\dot{\theta}(t)$ (and hence $\phi$ and $\dot{\phi}$ ) are directly available from measurement, and enter the control input $u$ without delay. Thus, applying a simple proportional-plus-derivative (PD) control, we consider the model

$$
\begin{array}{r}
m l^{2} \ddot{\phi}+b \dot{\phi}+b \omega+m l\left[g-\omega^{2} p \sin (\omega t)\right] \sin (\phi+\omega t)= \\
=m l \operatorname{PD}\left[\phi-\phi_{0}\right]
\end{array}
$$

where

$$
\mathrm{PD}[x]=-G_{1} \cdot x-G_{2} \cdot \dot{x},
$$

$\phi_{0}(t)$ is the control demand signal (we call it the control target), and $G_{1}$ and $G_{2}$ are control gains. Let us assume that $\phi_{*}$ is a periodic orbit of the uncontrolled parametric pendulum (2). We observe the following properties of the controlled system (3):

1. [Non-invasiveness] If $\phi_{0}=\phi_{*}$ then $\phi_{*}$ is a periodic orbit of the controlled system (3).

2. [Stability] With an appropriate choice of control gains $G_{1}$ and $G_{2}, \phi_{*}(t)$ is a stable periodic orbit of (3), regardless of its stability in the uncontrolled system (2). Thus, if $\phi_{0}=\phi_{*}$ then the difference between output and input, $\phi_{0}(t)-\phi(t)$, approaches zero for $t \rightarrow \infty$ for any initial value $\left(\phi\left(t_{0}\right), \dot{\phi}\left(t_{0}\right)\right)$ of (3) near $\left(\phi_{0}\left(t_{0}\right), \dot{\phi}_{0}\left(t_{0}\right)\right)$.

An appropriate choice for $G_{1}$ and $G_{2}$ in the idealized system (3) is, for example, $G_{2}=G_{1} / 2=G$ where $G$ is sufficiently large (in a practical implementation the size of $G$ is restricted by the control loop latency and the magnitude of disturbances). Our particular choice of gains is not optimal but our arguments are valid for any set of gains stabilizing $\phi_{*}$.

3. [Smoothness] If the control target $\phi_{0}$ is a periodic function near $\phi_{*}$ then the output of the controlled system (3) converges to a periodic function $\phi$. This asymptotic output $\phi$ depends (locally) uniquely on the control target $\phi_{0}$. Moreover, the asymptotic output $\phi$ depends smoothly on the input $\phi_{0}$.

These three observations make it possible to define a nonlinear map $M_{\infty}$ mapping a neighborhood of a periodic orbit $\phi_{*}$ of (2) in the space $C_{\text {per }}([0,2 \pi / \omega] ; \mathbb{R})$ of continuous periodic functions on the interval $[0,2 \pi / \omega]$ back to itself:

$$
M_{\infty}: U\left(\phi_{*}\right) \subset C_{\mathrm{per}}([0,2 \pi / \omega] ; \mathbb{R}) \mapsto C_{\mathrm{per}}([0,2 \pi / \omega] ; \mathbb{R}) .
$$

Evaluating $M_{\infty}$ in a point $\phi_{0} \approx \phi_{*}$ works as follows: $\phi_{0}$ is a periodic function with period $2 \pi / \omega$. Insert $\phi_{0}(t)$ as periodic control target into the PD control law (4) and observe the output of the controlled system (3). Since the controlled system is stable, after the transients have died down the output converges to some periodic function $\phi(t)$, which has also period $2 \pi / \omega$. The value of the map $M_{\infty}$ in $\phi_{0}$ is defined as this periodic output $\phi: M_{\infty}\left(\phi_{0}\right)=\phi$. The observation about smoothness guarantees that the map $M_{\infty}$ is differentiable with respect to its argument $\phi_{0}$.

The non-invasiveness of the feedback control states that $\phi_{*}$ is a fixed point of $M_{\infty}$. Moreover, if $\phi_{*}$ is a regular periodic orbit of (2) (that is, no Floquet multiplier of $\phi_{*}$ is equal to 1) then $\phi_{*}$ is a regular fixed point of $M_{\infty}$ (that is, the Fréchet derivative of $M$, [ $\left.\partial / \partial \phi_{0}\right] M$, in $\phi_{0}=\phi_{*}$ has no eigenvalue 1). Consequently, one can find regular periodic orbits (regardless of their stability) of the uncontrolled system (2) by applying a Newton iteration to the fixed point problem

$$
M_{\infty}\left(\phi_{0}\right)=\phi_{0},
$$

which only requires observations of the controlled system (3).

\section{Applying time-delayed feedback control}

A difficulty in the solution of the fixed point problem (6) is that the Newton iteration requires an approximation of the Fréchet derivative (the infinite-dimensional Jacobian) $J_{\infty}=\left[\partial / \partial \phi_{0}\right] M_{\infty}$ of $M_{\infty}$. This Jacobian $J_{\infty}$ is, in general, not known analytically so that it has to be estimated, for example, by finite differences. If the argument of $M_{\infty}, \phi_{0}$, is discretized by projecting it onto its $N$ leading Fourier modes then each evaluation of $J_{\infty}$ requires running the controlled experiment (3) at least $2 N+2$ times. This may be too timeconsuming to be practical in a mechanical experiment because the transients may take a few seconds to die down for each tested control target $\phi_{0}$. This problem can be tackled by estimating the Jacobian using a Broyden update [8], as suggested in [20]. This works well in simulations of the parametric pendulum (using (2)) and other systems where the expected solution is only moderately non-harmonic (such that the number of Fourier modes $N$ can be chosen as a small number) but has not been tested in experiments. The alternative, employed in [19], is the introduction of time-delayed 
feedback [17]. This reduces the infinite-dimensional fixedpoint problem (6) to a one-dimensional fixed point problem for a scalar variable, the average of $\phi$ over the last period. If we define

$$
\begin{aligned}
\operatorname{avg}[\phi](t) & =\frac{\omega}{2 \pi} \int_{0}^{2 \pi / \omega} \phi(t-\tau) \mathrm{d} \tau \\
\phi_{0}(t) & =\phi(t-2 \pi / \omega)+\tilde{\phi}_{0}-\operatorname{avg}[\phi](t-2 \pi / \omega),
\end{aligned}
$$

and insert this recursively defined $\phi_{0}$ into the feedback loop (3) then only the scalar quantity $\tilde{\phi}_{0}$ is an unknown additional parameter, rather than the whole (infinite-dimensional) periodic control target $\phi_{0}$. We note that avg $[x]$ is the average of a function $x$ of time over the past period of excitation. Whenever $\phi$ is periodic the quantity avg $[\phi]$ is a constant. (Hence, $\operatorname{avg}[x]$ corresponds to Fourier mode number zero of $x$ if $x$ is periodic.) If we insert $\phi_{0}(t)$ as defined by (7) for our control target into the system with control (3) then we observe the following. If $\phi_{*}$ is a periodic orbit of the uncontrolled system (2), and we choose the scalar parameter $\tilde{\phi}_{0}$ equal to $\operatorname{avg}\left[\phi_{*}\right]$, then $\phi_{*}$ is also a periodic orbit of the time-delayed system (3), (7). If $\phi_{*}$ is a dynamically stable periodic orbit of the time-delayed system (3), (7) then it makes sense to define the nonlinear map $M_{1}$, mapping a neighborhood of $\operatorname{avg}\left[\phi_{*}\right]$ in $\mathbb{R}$ back to itself, as:

$$
M_{1}: U\left(\operatorname{avg}\left[\phi_{*}\right]\right) \subset \mathbb{R} \mapsto \mathbb{R}, M_{1}\left(\tilde{\phi}_{0}\right):=\lim _{t \rightarrow \infty} \operatorname{avg}[\phi](t)
$$

where $\phi$ is the output of the controlled system (3), (7) when we insert the scalar $\tilde{\phi}_{0}$ into (7). Thus, whenever the map $M_{1}$ is well-defined we can find periodic orbits of the uncontrolled system (2) also as fixed points of $M_{1}$ instead of $M_{\infty}$.

However, it is not immediately clear if $\phi_{*}$ is a stable periodic orbit of the time-delayed system (3), (7) when we pick $\tilde{\phi}_{0}=$ $\operatorname{avg}\left[\phi_{*}\right]$ (even though $\phi_{*}$ is stable as a periodic orbit of (3) if the fixed periodic control target $\phi_{0}$ equals $\phi_{*}$ ). We observed in experiments [19] that $\phi_{*}$ is indeed stable in (3), (7) regardless of its stability in the uncontrolled system (2). However, it is not obvious why this is the case: (7) is a modification of the classical time-delayed feedback scheme introduced initially in [17] (which simply chooses $\phi_{0}(t)=\phi(t-2 \pi / \omega)$ ). Timedelayed feedback does not converge uniformly near folds of periodic orbits of the uncontrolled system (2) (and we study the rotations of the parametrically excited pendulum near a fold). The following two subsections study the properties of the feedback-controlled system (3), (7) numerically and analytically.

\subsection{Convergence of time-delayed feedback for large frequencies}

In this section we show that the controlled system (3) combined with the choice of control target as defined by (7) converges for large frequency $\omega$. Furthermore, we show that in this high-frequency regime the convergence rate is identical up to terms of order $\omega^{-1 / 2}$ to the convergence rate of the classical feedback control where one inserts a fixed periodic function $\phi_{0}$ into (3). For the experimental setting in Section 4 frequencies larger than $2 \mathrm{~Hz}$ can be considered as large.

In the large frequency regime periodic orbits of (2) are born approximately at $p=2 b /(\omega m l)$ in a saddle-node bifurcation [21]. Moreover, at this saddle-node $\phi(t)$ satisfies $|\phi(t)-\operatorname{avg}[\phi]| \ll 1$. This suggests that the excitation amplitude $p$ is typically small in length (of order $\omega^{-1}$ ) but the force exerted by the excitation is still large (of order $\omega$ ), implying a natural scaling of $p \sim \omega^{-1}$. The control force is of the same order as the force exerted by the excitation if the gains the scale as $G_{1} \sim \omega$ and $G_{2} \sim \sqrt{\omega}$ (because they are factors in the control force as introduced in (4)). Thus, we introduce scaled parameters

$$
a \sim-\omega p, g_{1} \sim \omega^{-1} G_{1} \text {, and } g_{2} \sim \sqrt{\omega^{-1}} G_{2}
$$

and assume that $a, g_{1}$ and $g_{2}$ are all of order 1, independent of $\omega$. Using the scaled parameters, the non-dimensionalized equation for the parametrically excited pendulum with timedelayed feedback control as defined by (7) has the form

$$
\begin{aligned}
& \quad \ddot{\phi}+\gamma \dot{\phi}+\gamma \omega+[1+a \omega \sin (\omega t)] \sin (\phi+\omega t)= \\
& -\omega g_{1}[\phi-\phi(t-2 \pi / \omega)]-\sqrt{\omega} g_{2}[\dot{\phi}-\dot{\phi}(t-2 \pi / \omega)] \\
& -\omega g_{1}\left[\operatorname{avg}[\phi](t-2 \pi / \omega)-\tilde{\phi}_{0}\right]-\sqrt{\omega} g_{2}[\operatorname{avg}[\dot{\phi}](t-2 \pi / \omega)]
\end{aligned}
$$

In (10) we have dropped the time argument $t$ from $\phi$ if it comes without delay, only keeping the delayed terms expressly as arguments. The relationship between the original quantities and the parameters in (10) is given by

$$
\begin{aligned}
& \gamma=\frac{b}{m \sqrt{g l^{3}}}, \quad \omega_{\text {new }}=\frac{\omega_{\text {old }}}{\sqrt{g / l}}, \quad t_{\text {new }}=t_{\text {old }} \sqrt{g / l} \\
& a=-\omega_{\text {new }} \frac{p}{l}, \quad g_{1}=\frac{G_{1}}{g \omega_{\text {new }}}, \quad g_{2}=\frac{G_{2}}{\sqrt{g l \omega_{\text {new }}}} .
\end{aligned}
$$

We introduce the small parameter $\epsilon=\sqrt{\omega^{-1}}$ and rescale time again by $\epsilon$ such that the new time is $t_{\text {new }}=t_{\text {old }} / \epsilon$ :

$$
\begin{aligned}
\ddot{\phi}+\epsilon \gamma & \dot{\phi}+\gamma+\left[\epsilon^{2}+a \sin (t / \epsilon)\right] \sin (\phi+t / \epsilon)= \\
= & -g_{1}[\phi-\phi(t-2 \pi \epsilon)]-g_{2}[\dot{\phi}-\dot{\phi}(t-2 \pi \epsilon)] \\
& -g_{1}\left[\operatorname{avg}_{\epsilon}[\phi](t-2 \pi \epsilon)-\tilde{\phi}_{0}\right]-g_{2}\left[\operatorname{avg}_{\epsilon}[\dot{\phi}](t-2 \pi \epsilon)\right]
\end{aligned}
$$

where $\operatorname{avg}_{\epsilon}[x]$ is defined by

$$
\operatorname{avg}_{\epsilon}[x](t)=\frac{1}{2 \pi \epsilon} \int_{0}^{2 \pi \epsilon} x(t-\tau) \mathrm{d} \tau .
$$

Despite the large coefficient occurring in $\operatorname{avg}_{\epsilon}$ the norm of the operator $\operatorname{avg}_{\epsilon}$ is 1, independently of $\epsilon$ in the space $C^{k}$ (the space of $k$ times continuously differentiable functions). Consequently, equation (12) is in the standard form for averaging [11]. All coefficients are at most of order 1 (including their Lipschitz constants) and the time-dependent coefficients are periodic with a period of $2 \pi \epsilon$. A near-identity transformation of the form $(\phi, \dot{\phi})=\left(\phi_{\mathrm{av}}, \dot{\phi}_{\mathrm{av}}+\epsilon \eta\left(\phi_{\mathrm{av}}, \dot{\phi}_{\mathrm{av}}, t / \epsilon, \epsilon\right)\right)$ transforms (12) into (we drop the index av from the transformed quantities)

$$
\ddot{\phi}+\gamma+\frac{a}{2} \cos \phi=-g_{1}\left[\phi-\tilde{\phi}_{0}\right]-g_{2} \dot{\phi}+\epsilon r(\phi, \dot{\phi}, t / \epsilon, \epsilon)
$$

where the remainder $r$ and the transformation $\eta$ have period $2 \pi \epsilon$ in $t$ and contain terms depending on the history of $\phi$ and $\dot{\phi}$ on the interval $[t-4 \pi \epsilon, t]$. The only important property of $r$ is that its norm as a function in $C$ (the space of continuous 
functions) is bounded uniformly for small $\epsilon$ if $\phi$ and $\dot{\phi}$ are bounded as functions in $C^{1}$ (the space of continuously differentiable functions). This is guaranteed because the linear expressions

$$
\begin{aligned}
& L_{d}: x(\cdot) \mapsto x(\cdot)-x(\cdot-2 \pi \epsilon) \\
& L_{a}: x(\cdot) \mapsto \operatorname{avg}_{\epsilon}[x](\cdot-2 \pi \epsilon)-x(\cdot)
\end{aligned}
$$

satisfy

$$
\begin{aligned}
& \max _{t \in[-2 \pi \epsilon, 0]}\left|L_{d}[x](t)\right| \leq 2 \pi \epsilon \max _{t \in[-4 \pi \epsilon, 0]}|\dot{x}| \\
& \max _{t \in[-2 \pi \epsilon, 0]}\left|L_{a}[x](t)\right| \leq 4 \pi \epsilon \max _{t \in[-4 \pi \epsilon, 0]}|\dot{x}|
\end{aligned}
$$

This implies that the terms $L_{d}[\phi](t), L_{d}[\dot{\phi}](t)$, present in (12), are both of order $\epsilon$ and can be incorporated into the remainder $\epsilon r$ in (13). Similarly, the terms $\operatorname{avg}_{\epsilon}[\phi](t-2 \pi \epsilon)$ and $\operatorname{avg}_{\epsilon}[\dot{\phi}](t-2 \pi \epsilon)$, both present in (12), can be approximated by $\phi(t)$ and $\dot{\phi}(t)$, respectively, where the error again can be incorporated into the remainder term $\epsilon r$.

The transformation from (12) to (13) does not involve any approximation: (12) and (13) are equivalent. The averaging technique is only formally developed for ODEs in textbooks such as [11]. Textbook [11] reduces the statement about the validity of averaging to a theorem about the persistence of normally hyperbolic manifolds, for which [11] refers to [12, 9] for ODEs. However, such a statement is also true in more general situations, in particular, for semiflows generated by delay differential equations (DDEs) $[2,3]$. We note that the time- $4 \pi \epsilon$ map $T_{\epsilon}$ (the second iterate of the stroboscopic map), generated by (13), maps initial values $\left(\phi_{\text {ini }}, \dot{\phi}_{\text {ini }}\right) \in C^{1}\left([-4 \pi \epsilon, 0] ; \mathbb{R}^{2}\right)$ at time $t=0$ back into $C^{1}\left([-4 \pi \epsilon, 0] ; \mathbb{R}^{2}\right)$ at time $t=4 \pi \epsilon$ (that is, the solution of (13) with initial value $\left(\phi_{\text {ini }}, \dot{\phi}_{\text {ini }}\right)$ is continuously differentiable on the interval $[0,4 \pi \epsilon])$. Moreover, $T_{\epsilon}$ is a perturbation of order $\epsilon$ of the map $T_{0}$ (obtained by setting $\epsilon=0$ in (13)) generated by

$$
\ddot{\phi}+\gamma+\frac{a}{2} \cos \phi=-g_{1}\left[\phi-\tilde{\phi}_{0}\right]-g_{2} \dot{\phi},
$$

which no longer depends on time explicitly and also involves no time delays. We note that in (14) the time-delayed feedback control has been reduced to a classical PD control of the form $-g_{1}\left[\phi-\tilde{\phi}_{0}\right]-g_{2} \dot{\phi}$ for a fixed point $\left(\phi_{*}, 0\right)$ of $\ddot{\phi}+\gamma+\frac{1}{2} a \cos \phi=0$.

Consequently, for large $\omega$ and the parameter scaling (9), the uncontrolled system has one periodic orbit of saddle type satisfying $\operatorname{avg}[\phi]=\pi-\arccos (2 \gamma / a)+O(\epsilon)$ and one periodic orbit of node type (its stability is of order $\epsilon=\sqrt{1 / \omega}$ ) satisfying $\operatorname{avg}[\phi]=\pi+\arccos (2 \gamma / a)+O(\epsilon)$ for $a>2 \gamma$. Approximation (14) shows that time-delayed feedback control of the form (7) stabilizes the saddle periodic orbit for large forcing frequencies $\omega$ if

$$
g_{1}>\frac{1}{2} \sqrt{a^{2}-4 \gamma^{2}}, \quad g_{2}>0 .
$$

\subsection{Convergence of time-delayed feedback for frequencies of order 1}

Figure 1 shows the curve in the two-parameter $\left(p, \tilde{\phi}_{0}\right)$-plane that is implicitly defined by the following scalar condition: the controlled system with time-delayed feedback (3), (7) has a periodic orbit $\phi\left[\tilde{\phi}_{0}, p\right](t)$ that satisfies $\operatorname{avg}[\phi]=\tilde{\phi}_{0}$. These

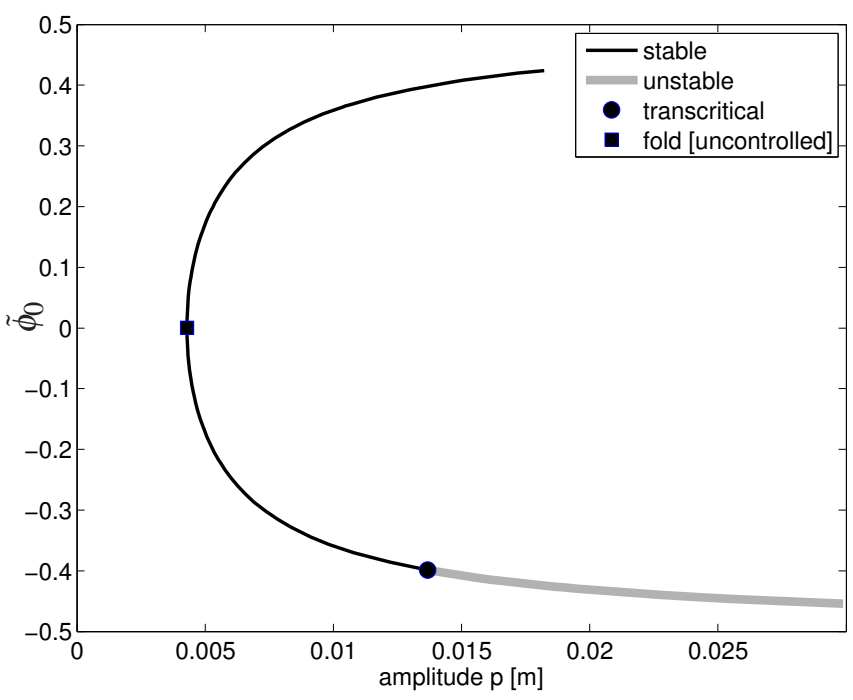

Figure 1: Loss of stability for the controlled system at frequency $\omega=3 \mathrm{~Hz}$, gain $G_{1}=1 \mathrm{~m} \mathrm{~s}^{-2}$ and $G_{2}=$ $0.5 \mathrm{~m} \mathrm{~s}^{-1}$ in (3), (4), (7). Note that te uncontrolled pendulum (2) loses its stability already at the fold point. The fixed parameters were set to values corresponding to the experimental setup discussed in Section 4: $m=0.05 \mathrm{~kg}, l=0.2772 \mathrm{~m}, b=5.565 \times$ $10^{-4} \mathrm{~kg} \mathrm{~m}^{2} \mathrm{~s}^{-1}$.

periodic orbits are identical to periodic orbits of the uncontrolled system (2). Numerical computation of the Floquet multipliers shows that for all parameter pairs $\left(p, \tilde{\phi}_{0}\right)$ in the vicinity of the stable (dark) part of the curve the periodic orbit $\phi\left[\tilde{\phi}_{0}, p\right](t)$ of the controlled system with time-delayed feedback (3), (7) is stable and satisfies $\operatorname{avg}[\phi] \approx \tilde{\phi}_{0}$. Thus, in this region the map $M_{1}$ can be defined as given in (8).

At the transcritical point (filled circle in Fig. 1) the periodic orbit $\phi\left[\tilde{\phi}_{0}, p\right](t)$ still exists (and satisfies avg $[\phi]=\tilde{\phi}_{0}$ in this point) but it loses its stability: one Floquet multiplier passes through 1. While the location of the transcritical point depends on the gains $G_{1}$ and $G_{2}$, the location of the curve itself is independent of the gains. (We chose $G_{1}=1 \mathrm{~m} \mathrm{~s}^{-2}$, $G_{2}=0.5 \mathrm{~m} \mathrm{~s}^{-1}$ for the numerical computation of the stability along the curve in Fig. 1.)

Without control the upper part of the curve corresponds to dynamically stable rotations, and this branch loses its stability at the fold point corresponding to the minimal amplitude $p$ that supports rotation [21]. Consequently, applying time-delayed feedback, as defined in (7), stabilizes all saddle rotations of the uncontrolled system (2) successfully between the fold and the transcritical point in Fig. 1. In this region the definition (8) of the map $M_{1}$ makes sense. For large frequencies the location of this transcritical point is approximately given by relation (15). The general expression for the transcritical point is given implicitly by equation (2), extended by 


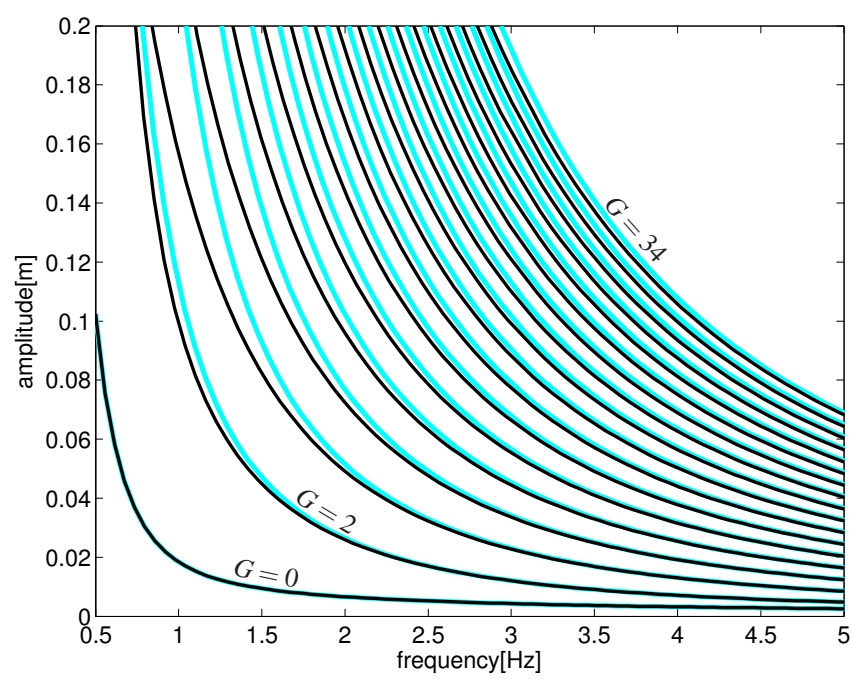

Figure 2: Loss of stability in the controlled system in the $(\omega, p)$-plane for varying control gains $G$. The gains $G$ vary from 0 to 34 in steps of 2 . The thin dark curves show when system (3), (4), when using classical PD control with the control target $\phi_{0}=\phi_{*}$, loses stability. The thick light curves show when system (3), (7) loses stability. Fixed parameters are (as estimated for the physical pendulum discussed in Section 4): $m=0.05 \mathrm{~kg}, l=0.2772 \mathrm{~m}, b=5.565 \times 10^{-4} \mathrm{~kg} \mathrm{~m}^{2} \mathrm{~s}^{-1}$.

its linearized problem and two integral conditions [18]:

$$
\begin{aligned}
0= & m l^{2} \ddot{\phi}+b \dot{\phi}+b \omega+ \\
& +m l\left[g-\omega^{2} p \sin (\omega t)\right] \sin (\phi+\omega t), \\
0= & m l^{2} \ddot{y}+b \dot{y}+ \\
& +m l\left[g-\omega^{2} p \sin (\omega t)\right] \cos (\phi+\omega t) y+m l G_{1} \tilde{y}_{0}, \\
0= & \frac{\omega}{2 \pi} \int_{0}^{2 \pi / \omega} y \mathrm{~d} \tau-\tilde{y}_{0}, \\
0= & \frac{\omega}{2 \pi} \int_{0}^{2 \pi / \omega} y^{2}+\dot{y}^{2} \mathrm{~d} \tau-1,
\end{aligned}
$$

where $\phi$ and $y$ are periodic and $\tilde{y}_{0}$ is a scalar. Equation (16) defines $\phi$ as a periodic orbit of the uncontrolled system (and, thus, of (3), (7) with $\left.\tilde{\phi}_{0}=\operatorname{avg}[\phi]\right)$. Equation (17) defines $y$ as the eigenvector of the system with time-delayed feedback (3), (7) linearized in $\phi$. The eigenvector $y$ corresponds to the Floquet multiplier 1 (thus, Eq. (17) guarantees that system (3), (7) has indeed a Floquet multiplier 1). The scalar condition (18) defines the quantity $\tilde{y}_{0}$ (which enters (17)) as the average of $y(t)$, and condition (19) requires the norm of the eigenvector $y$ to be equal to 1 . The system (16)-(19) consists of two second-order periodic equations and two scalar equations for four periodic functions $(\phi, \dot{\phi}, y$ and $\dot{y})$ and one auxiliary scalar quantity $\tilde{y}_{0}$. Thus, system (16)-(19) defines a surface in the three-dimensional parameter $(\omega, p, G)$-space (we always choose $G_{1}=2 G_{2}=G$ in original units). Note that the linearization (17) does not contain any delayed terms because $y$ is the eigenvector corresponding to the Floquet multiplier 1.

Figure 2 shows the contour curves of this implicitly defined surface as light curves in the $(\omega, p)$-plane, for different fixed values of $G$ that increase in steps of size 2 from 0 to 34 . The lowest curve in Fig. 2 corresponds to $G=0$, and, thus, this transcritical bifurcation curve coincides with the curve where periodic orbits of the uncontrolled system (2) are born in a saddle-node bifurcation. For a given gain $G$ the map $M_{1}$ is well-defined for all values of $\omega$ and $p$ below the light curve corresponding to this choice of $G$. The dark curves in Fig. 2 show when classical feedback control loses its stability. They are defined by a modification of system (16)-(19): one replaces the term $m l G_{1} \tilde{y}_{0}$ by $m l\left[G_{1} y+G_{2} \dot{y}\right]$ (and drops condition (18)). Figure 2 shows that the system controlled with time-delayed feedback (3), (7) is able to stabilize the saddle-type rotations of (2) for the same choice of gains as when pure PD feedback control (3) is applied.

In summary, for our particular problem, finding saddletype rotations of the driven pendulum experimentally, timedelayed feedback of the form (7) is a sensible choice because it stabilizes periodic orbits for the same gains as classical feedback control. The advantage is that time-delayed feedback introduces only a scalar parameter, $\tilde{\phi}_{0}$, that has to be solved for by Newton iteration as a fixed point of the map $M_{1}$ defined in (8).

\subsection{Embedding into pseudo-arclength continuation}

Two difficulties in finding periodic orbits as fixed points of $M_{1}$ are:

- The Newton iteration typically requires a good initial guess to have guaranteed success, and

- at special parameter values of $p$ (the excitation amplitude) the fixed point $\tilde{\phi}_{*}$ of the map $M_{1}$ is singular. This happens exactly at the minimal amplitude $p$ that still supports periodic rotations $\phi$, which is a saddle-node (one Floquet multiplier is 1 in the uncontrolled system) of the system.

These difficulties can be overcome by embedding the fixed point problem for $M_{1}$ into a pseudo-arclength continuation $[14,6]$. If one is able to vary a system parameter, in our case the excitation amplitude $p$ in (3), then one can treat the parameter also as an unknown variable. The map $M_{1}$ depends on $p$ such that we have to include it as anditional argument, writing $M_{1}\left(\tilde{\phi}_{0}, p\right)$. The dependence of $M_{1}$ on $p$ is smooth. The set of fixed points of $M_{1}$, satisfying $M_{1}\left(\tilde{\phi}_{0}, p\right)=\tilde{\phi}_{0}$, defines a curve of points $\left(\tilde{\phi}_{0}, p\right)$ in the plane $\mathbb{R}^{2}$. Assume that we have found an initial point on this curve. In the experiment described in Section 4 we easily discover a stable periodic orbit $\phi_{*}$ for some sufficiently large $p$ (for example, $p \approx 2 \mathrm{~cm}$ at $\omega=3 \mathrm{~Hz}$ ). Then we can track the curve starting from this initial point by repeatedly solving the following two-dimensional system:

$$
\begin{aligned}
\tilde{\phi}_{0} & =M_{1}\left(\tilde{\phi}_{0}, p\right) \\
h & =\tilde{\phi}_{\tan }^{T}\left(\tilde{\phi}_{0}-\tilde{\phi}_{\text {old }}\right)+p_{\text {tan }}^{T}\left(p-p_{\text {old }}\right) .
\end{aligned}
$$

In (20), (21), $\tilde{\phi}_{0}$ and $p$ are the variables to be solved for, $\left(\tilde{\phi}_{\text {old }}, p_{\text {old }}\right)$ is the point found previously on the curve, $\left(\tilde{\phi}_{\tan }, p_{\tan }\right)$ is an approximation of the tangent of unit length to the curve in the previous point ( $\left.\phi_{\text {old }}, p_{\text {old }}\right)$, and $h$ is a small quantity approximating the stepsize along the implicitly defined curve. One possible approximation for the unit tangent 


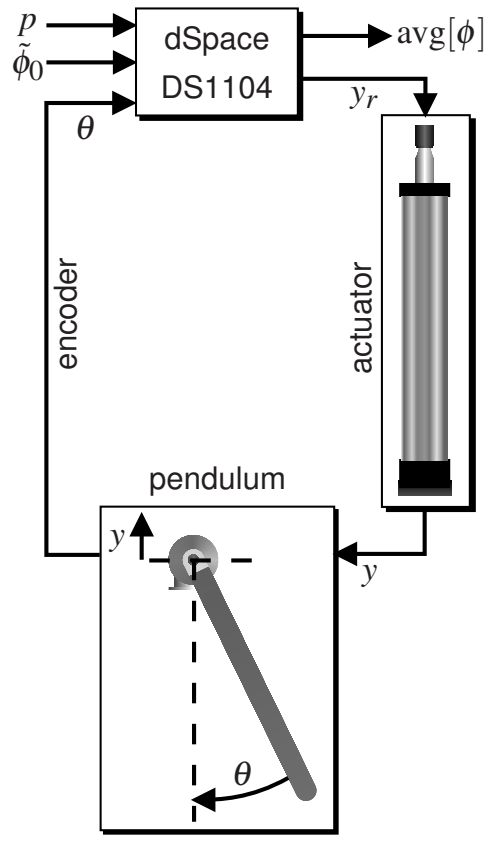

(a)

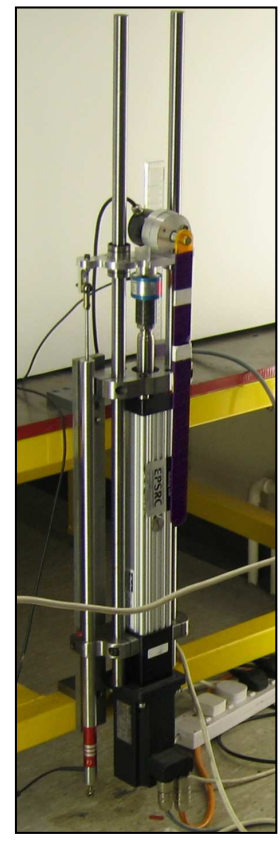

(b)
Figure 3: Experimental setup: (a) sketch, (b) photo showing the pendulum and the actuator. The dSpace board obtains $\phi=\theta-\omega t$ and outputs avg $[\phi]$ and $y_{r}$ by computing recursion (7), the ODEs (22) and (27), and the reset law (26) in real-time. The inputs $p$ and $\tilde{\phi}_{0}$ are piecewise constant and obtained by recursion (29). See Fig. 7 for a typical time profile of inputs and outputs.

is the secant through the previous two points along the curve. (The choice of tangent for the initial step along the curve can be $(0, \pm 1)$ because the initial fixed point $\tilde{\phi}_{*}$ is regular.)

Equation (21) is called the pseudo-arclength condition [6]. Solving a sequence of extended systems of the form (20), (21) to obtain a sequence of solutions $\left(\tilde{\phi}_{0, k}, p_{k}\right)$ on the curve defined by $M_{1}\left(\tilde{\phi}_{0}, p\right)=\tilde{\phi}_{0}$ alleviates both difficulties of the fixedpoint approach: in each step along the curve a good initial guess for the Newton iteration is $\left(\tilde{\phi}_{0}, p_{0}\right)=\left(\tilde{\phi}_{\text {old }}+h \tilde{\phi}_{\text {tan }}, p_{\text {old }}+\right.$ $\left.h p_{\tan }\right)$. Also, even if $\left[\partial / \partial \tilde{\phi}_{0}\right] M_{1}$ is singular, the Jacobian of the extended system $(20),(21)$ is regular as long as $[\partial / \partial p] M_{1}$ is not zero simultaneously. In particular, at saddle-nodes (folds with respect to the continuation parameter) the extended system is known to be regular. Extension (21) minimizes the condition number of the linearization if $\left(\tilde{\phi}_{\tan }, p_{\tan }\right)$ is exactly the unit tangent [6].

\section{Experimental implementation}

The experimental setup differs from the idealized model (3) in several aspects; see Fig. 3(a) for a sketch of the setup and Fig. 3(b) for a photo. First, control can be applied only as part of the vertical pivot motion $y(t)$, which is driven by a servomechanical actuator. That is, one can prescribe a demand signal $y_{r}(t)$, and the position controller driving the actuator aims to guarantee that the actuator motion $y(t)$ tracks this reference signal. Typically, the actuator achieves tracking with a small delay: $y(t)=y_{r}(t-\tau)$ with $\tau<20 \mathrm{~ms}$ is a good model for the transfer system to the actuator. Thus, control enters the experimental setup by feeding a reference signal given by the ODE

$$
\ddot{y}_{r}(t)=-\omega^{2} p \sin (\omega t)+u(t)
$$

into the actuator. A digital incremental encoder provides measurements of the angle $\theta$ (which can be converted to $\phi=\theta-\omega t$ ) as output of the experiment, and an LVDT displacement transducer tracks the actuator movement $y(t)$ (which is only used in the internal position controller of the actuator). Using the output $\phi$ (or $\theta$ ) we apply feedback control defined by:

$$
u(t)=S(\theta(t)) \operatorname{PD}\left[\phi(t)-\phi_{0}(t)\right]
$$

where $\phi_{0}$ is given by recursion (7), the gains are chosen $G_{1}=$ $1 \mathrm{~m} \mathrm{~s}^{-2}, G_{2}=0.5 \mathrm{~m} \mathrm{~s}^{-1}$, and $S$ is a cut-off function given by

$$
S(\theta)= \begin{cases}{[\sin \theta]^{-1}} & \text { if }|\sin \theta|>0.2, \\ 0 & \text { otherwise. }\end{cases}
$$

Using this cut-off function $S$ the feedback control applied to (22), (23) is only equal to the idealized control present in (3) for a part of the rotation: $S$ cancels the term $\sin (\phi+\omega t)=$ $\sin \theta$ that is multiplied with $\ddot{y}$ as long as the angle $\theta$ is not close to 0 or $\pi$ (that is, the pendulum is not pointing up or down), except for the small delay in the transfer system. If the pendulum is rotating (nearly) periodically it spends only short periods near $\phi=0$ and $\phi=\pi$, which ensures local feedback stabilizability of periodic rotations in the classical sense [16]. Since $\theta=0$ (pendulum hanging down) is a coexisting stable steady state of the pendulum for frequencies greater than $2 \mathrm{~Hz}$ the feedback control (23) cannot stabilize the rotations globally, which is in contrast to the idealized control (3). However, in the context of the pseudo-arclength continuation (20), (21) corrections between successive control targets $\tilde{\phi}_{0}$ are always small, and the state $(\phi, \dot{\phi})$ of the experiment is always in the vicinity of the control target, making the control $u(t)$ small, and local feedback stabilization sufficient.

The size of permissible control signals $u$ in (22) is also restricted by the physical maximal amplitude of the actuator, imposing the restriction

$$
\left|y_{r}(t)\right|<0.03 \mathrm{~m} \text {. }
$$

The quantity $y_{r}$ as defined by (22) is itself not stable, and, thus, will not satisfy the restriction (25). In the worst case one would have to design a feedback control for the coupled system (2), (22) such that $u$ stabilizes both, $y_{r}$ and rotations of $\phi$, simultaneously. However, we can exploit the fact that for $\theta=0$ and $\theta=\pi$ corrections of $y$ do not influence the inclination angle $\theta$ of the pendulum (and, hence, $\phi$ ). Thus, we define $y_{r}$ as the solution of (22) but reset $\dot{y}_{r}$ according to the rule

$$
\dot{y}_{r} \mapsto-\frac{4 \pi}{\omega} y_{r} \quad \text { when } \sin \theta \text { changes its sign. }
$$

The resulting peaks in $\ddot{y}$ do not affect the rotating angle $\phi$ because its pre-factor $\sin \theta$ is close to zero.

The final modification compared to the idealized control (4) is that we approximate the angular velocity $\dot{\phi}$ in the PD control by a linear filter $\phi_{\nu}$ :

$$
\phi_{v}=\left(\phi-\phi_{f}\right) / h_{f}, \quad \dot{\phi}_{f}=\left(\phi-\phi_{f}\right) / h_{f}
$$


where $h_{f}$ is a small stepsize ( $h_{f}=0.01 \mathrm{~s}$ in the experiment), which is still large compared to the sampling time of the experiment and the stepsize of the real-time integration of (27).

Altogether, the numerical computation of the recursion (7), the linear second-order ODE (22) with the reset law (26), and the linear filter (27) have to be run in real-time in parallel to the experiment. This was achieved on a dSpace DS1104 RD real-time controller board. The sampling interval for realtime computations and measurements was $10^{-3} \mathrm{~s}$. (All instrumentation was identical to the setup presented in [10].) The continuation described in Section 3.3 was performed using the following procedure (described for the experimental run with fixed frequency $\omega=4 \mathrm{~Hz}$ ):

1. [Initial rotation] Initially we choose a large forcing amplitude of $p=2 \mathrm{~cm}$, set the control gain $G$ to zero, and swing up the pendulum. The stable rotation with period of the forcing has a sufficiently large basin of attraction at this amplitude and frequency, such that it can be found manually in this way.

2. [Initialization of recursion] After the transients have settled we measure the periodic output $\phi(t)$ and use this output to initialize the value of $\tilde{\phi}_{0}=\operatorname{avg}[\phi]$ and the history of $\phi_{0}$ in recursion (7) defining the time-delayed feedback terms. After initialization of $\phi_{0}$ the gains are set to their original values $\left(G_{1}=1 \mathrm{~m} \mathrm{~s}^{-2}, G_{2}=0.5 \mathrm{~m} \mathrm{~s}^{-1}\right)$.

3. [Quasi-Newton iteration] The equations (20), (21) define a two-dimensional system of equations of the form $F(y)=0$, which is solved by a relaxed Quasi-Newton recursion; see [20] for details. The variable $y$ consists of $p$ (in units of $0.05 \mathrm{~m}$, thus, $p$ is scaled to order 1 ) and $\tilde{\phi}_{0}$ (in radiant), and $F$ is given by

$$
\begin{aligned}
& F_{1}\left(p, \tilde{\phi}_{0}\right)=M_{1}\left(\tilde{\phi}_{0}, p\right) \\
& F_{2}\left(p, \tilde{\phi}_{0}\right)=\tilde{\phi}_{\tan }^{T}\left(\tilde{\phi}_{0}-\tilde{\phi}_{\text {old }}\right)+p_{\text {tan }}^{T}\left(p-p_{\text {old }}\right)-h .
\end{aligned}
$$

We choose a Quasi-Newton iteration with Broyden's rankone update:

$$
\begin{aligned}
y_{k+1} & =y_{k}-c_{y} J_{k}^{-1} F\left(y_{k}\right), \\
J_{k+1} & =J_{k}+c_{J}\left[\Delta_{k}-J_{k} \delta_{k}\right] \delta_{k}^{T} /\left(\delta_{k}^{T} \delta_{k}\right), \\
\Delta_{k} & =F\left(y_{k}\right)-F\left(y_{k-1}\right), \quad \delta_{k}=y_{k}-y_{k-1},
\end{aligned}
$$

where the parameters $c_{J}=0.7$ and $c_{y}=0.2$ are relaxation parameters (values closer to zero increase robustness but slow down convergence). We stop the iteration (29) if the residuals $F\left(y_{k}\right)$ and corrections $\delta_{k}$ are less than $5 \times 10^{-3}$ in modulus, and at least 4 iterations have been performed. The stepsize $h$ along the curve in (21) is 0.01. The initial guess for the Quasi-Newton Jacobian is $J_{0}=\left[\begin{array}{rr}-1 & 0 \\ 0 & 1\end{array}\right]$. The initial choice of $\left(p_{\text {old }}, \tilde{\phi}_{\text {old }}\right)$ are the values from the steps 1 and 2 . The initial approximation of the tangent vector $\left(p_{\tan }, \tilde{\phi}_{\tan }\right)$ is $(-1,0)$.

4. [Evaluation of $M_{1}$ ] The iteration (29) prescribes a sequence of pairs of $p$ and $\tilde{\phi}_{0}$. For each of these pairs one has to evaluate $M_{1}$ as part of the evaluation of $F$ in (28). This is done by adjusting the parameter $\tilde{\phi}_{0}$ in (7) and the forcing amplitude $p$ to their new value without stopping the experiment. Due to the gradual nature of

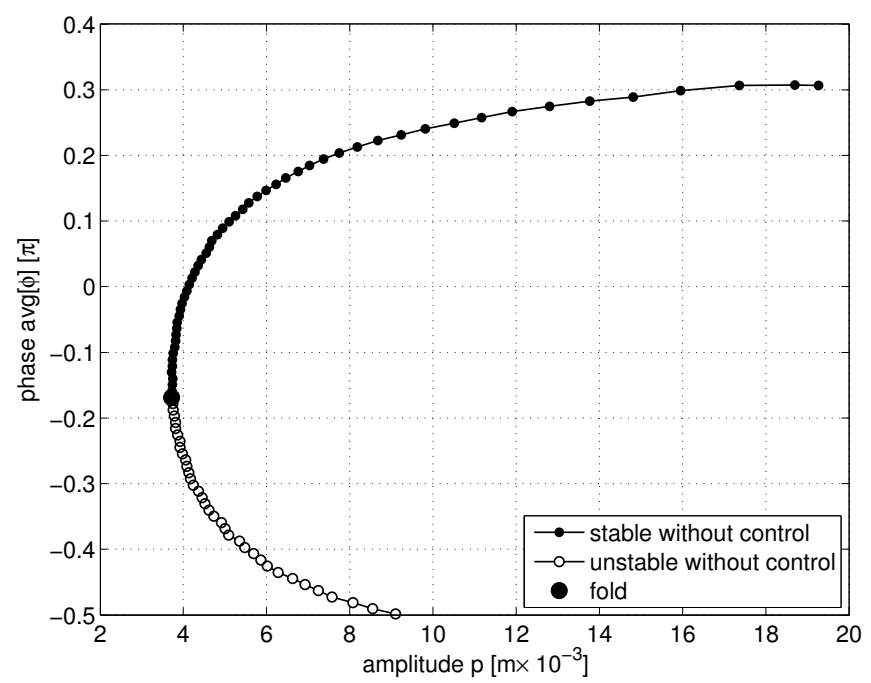

Figure 4: Experimental continuation for frequency $\omega=4 \mathrm{~Hz}$. The $y$-axis shows the average of $\phi$, which corresponds to the phase of the pendulum relative to the pivot motion. Choice of gains: $G_{1}=1 \mathrm{~m} \mathrm{~s}^{-2}, G_{2}=$ $0.5 \mathrm{~m} \mathrm{~s}^{-1}$; estimated parameters of the pendulum: $m=0.05 \mathrm{~kg}, l=0.2772 \mathrm{~m}, b=5.565 \times 10^{-4} \mathrm{~kg} \mathrm{~m}^{2} \mathrm{~s}^{-1}$.

the Quasi-Newton iteration and the continuation, these adjustments are always small (see Section 5 and Fig. 7). After a short transient the output $\phi$ of the experiment is again periodic. We accept the limit defining $M_{1}$ in (8) as converged if the $L^{2}$-norm of $\phi_{0}(t)-\phi_{0}(t-T)$ stays below $5 \times 10^{-3}$ for at least 3 periods.

The above procedure permits that the computations in the Quasi-Newton iteration (29) can be performed asynchronously in parallel to the experiment as shown in Fig. 3(a), placing no real-time constraints on (29). This becomes important if the number of unknowns in (29) is large. However, in our experiment the computational effort in (29) is negligible. In fact, the recursion (29) has been implemented in Simulink and downloaded onto the dSpace controller board (and is, thus, actually performed in real-time). Ref. [1] demonstrated that it is possible (and, indeed, more practical) to perform the Quasi-Newton iteration (29) asynchronous to the real-time computations.

\section{Experimental results}

Figures 4 and 5 show the results of the procedure described in Section 4 , where we fixed the frequency at $\omega=4 \mathrm{~Hz}$. At $p \approx$ $2 \mathrm{~cm}$ we find a stable rotation $\phi(t)$ satisfying avg $[\phi] \approx 0.3 \pi$. We initialize $\tilde{\phi}_{0}$ to this value and then follow the steps 3 and 4 of our experimental procedure - tracing out the whole curve in Fig. 4 in one experimental run of approximately 20 minutes. Every circle on the curve shown in Fig. 4 corresponds to a pair $\left(p, \tilde{\phi}_{0}\right)$ for which the difference $\phi-\phi_{0}$ is smaller than the Quasi-Newton tolerance $5 \times 10^{-3}$. Thus, every circle is (within tolerance) a periodic rotation of the uncontrolled pendulum. Without control the upper part of the branch (filled circles) in Fig. 4 would be dynamically stable, and the lower part (open circles) would be dynamically unstable (of saddle type). The fact that $\operatorname{avg}[\phi]>0$ means that the pendulum points 


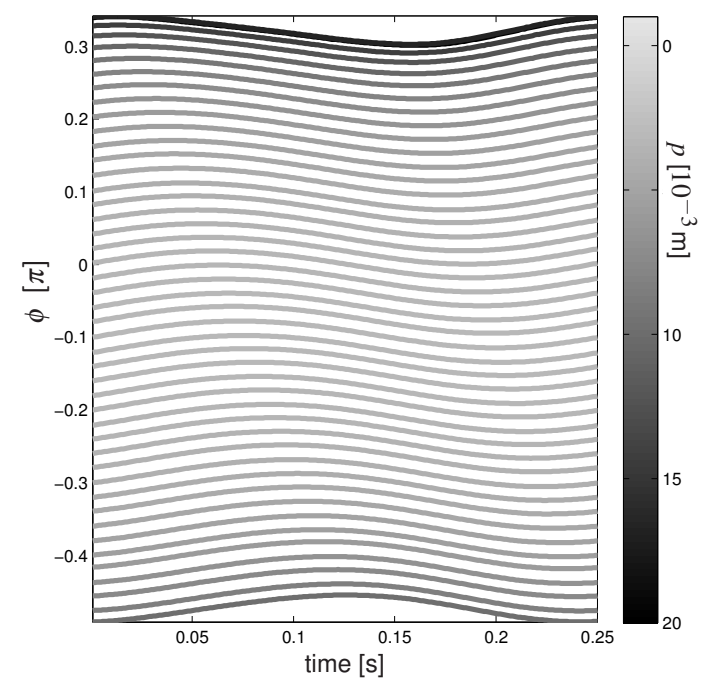

Figure 5: Time profiles $\phi(t)$ of recorded periodic rotations. Each profile corresponds to one circle in Fig. 4 (only every second solution is plotted); see caption of Fig. 4 for parameters.

upward when the pivot is near its top position during the forcing period for stable rotations. Close to the fold in Fig. 4 at $p_{\text {min }} \approx 3.5 \mathrm{~mm}$ the phase of the rotations changes rapidly to $\operatorname{avg}[\phi]<0$ : by approximately 60 degrees within $1 \mathrm{~mm}$ around $p_{\text {min }}$. The rapid change of the average of the solution profile implies that even a significant part of the dynamically stable part of the branch in Fig. 4 is practically inaccessible without feedback control [19]. Figure 5 shows the time profiles of $\phi(t)$ in the forcing period before the Quasi-Newton iteration was accepted as converged. Each profile corresponds to one circle in Fig. 4. The time profiles are all only moderately nonharmonic and the change is predominantly in their average $\operatorname{avg}[\phi]$ along the branch.

Figure 6 shows 10 branches of rotations, each continued in the forcing amplitude $p$, for fixed frequencies between $2 \mathrm{~Hz}$ and $5 \mathrm{~Hz}$. The branches are strictly ordered from larger to smaller forcing amplitudes for increasing frequency. Note that, for example, for $2 \mathrm{~Hz}$ the forcing amplitude is determined only up to an error of at least $0.2 \mathrm{~mm}$, a range within which the average of the rotation can change by up to 30 degrees. Still, the algorithm is able to follow the branch. In Fig. 6 there is an extreme difference between the scales of the axes because the changes in the artificial parameter $\tilde{\phi}_{0}$ are much larger than the changes of the forcing amplitude $p$. Hence, the apparently wiggly curves are nearly straight lines with uniform distances between successive points if the $x$-axis is rescaled by a factor 20 according to the scaling used in the procedure described in Section 4 . We also note that the loss of stability occurs a bit earlier than predicted in Fig. 2 for the idealized model. This is expected because this prediction used the stability in the exact fixed points of $M_{1}$ (where control is exactly zero), whereas the Quasi-Newton iteration also visits values of $\tilde{\phi}_{0}$ at small distances from the fixed points. Furthermore, due to the physical restriction (25) and the cut-off (24) disturbances cannot be corrected near the loss of stability of the feedback controlled experimental system.

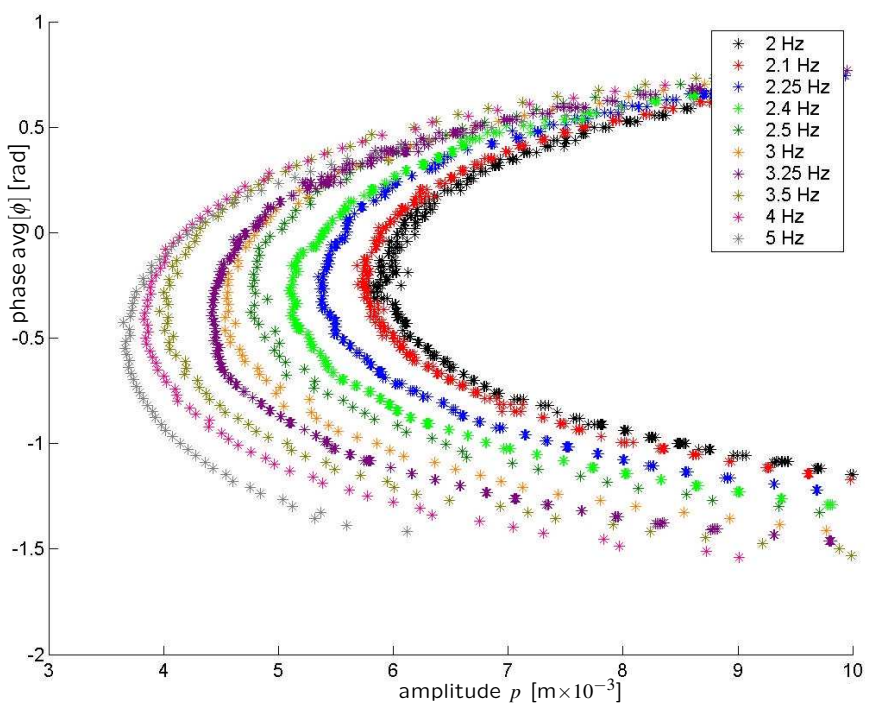

Figure 6: Overview of performed continuation runs in the forcing amplitude $p$ for frequencies ranging between $2 \mathrm{~Hz}$ and $5 \mathrm{~Hz}$. Continuations at smaller frequencies have a fold at higher amplitudes.

Figure 7(a) shows a $30 \mathrm{~s}$ window of the signal $\phi(t)-\phi_{0}(t)$ during the experimental continuation. This signal is the difference between the output $\phi$ and the result $\phi_{0}$ of the numerically computed recursion (7), which enters the control signal $u$ in (22): whenever the time profile $\phi-\phi_{0}$ shown in Fig. 7(a) is zero the control $u$ is zero, and we accept the current output as converged. This is indicated by filled circles along the time profile. The open circles along the time profile in Fig. 7(a) indicate times at which the time profile of $\phi_{0}$ is accepted as periodic. At these times $M_{1}$ is evaluated and the parameters $p$ and $\tilde{\phi}_{0}$ are changed according to the Quasi-Newton iteration (29). The corresponding time series of the parameter inputs are shown in the panels (b) and (c). Figure 7(a) shows that the control signal is quite small throughout the experiment, allowing the continuation to track the branch even though the feedback stabilizes rotations only locally. The higher peaks in the control signal in panel (a) come directly after convergence of the Quasi-Newton iteration. Then the continuation takes a new step along the branch, which means that the parameter values are updated to $\left(\tilde{\phi}_{0}, p_{0}\right)=\left(\tilde{\phi}_{\text {old }}+h \tilde{\phi}_{\text {tan }}, p_{\text {old }}+h p_{\text {tan }}\right)$, and this leads to initially larger control action. The Newton iteration then ensures in several steps that the correct periodic rotation is reached for which the control action is again (approximately) zero.

\section{Conclusion}

We have presented an experimental procedure that allows tracking of unstable periodic motions in nonlinear experiments that have a stabilizing feedback loop. The procedure converges uniformly even in the vicinity of singularities such as saddle-node bifurcations. Our approach also gives an estimate for the accuracy of the results: the product of residuals in the fixed point iteration of the map $M_{1}$ (which is measured) and the condition number of the Jacobian $J$ in the Quasi-Newton iteration (29) provide an upper bound for the 
(a)

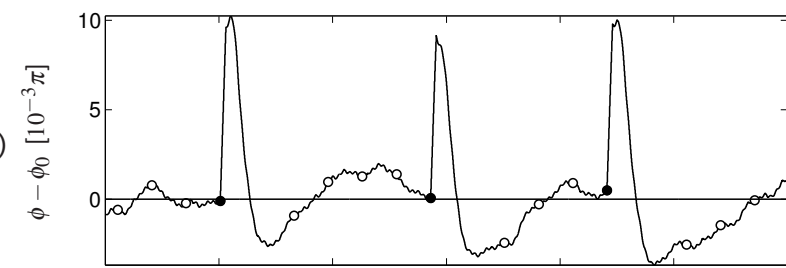

(b)

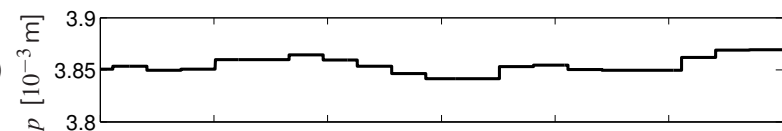

(c)

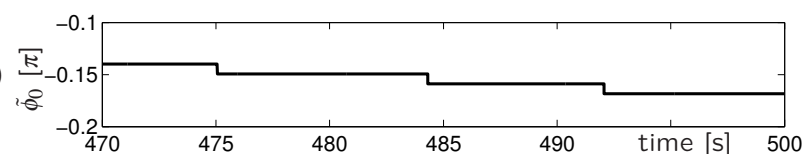

Figure 7: Panel (a) shows a short time window of the signal $\phi(t)-\phi_{0}(t)$, entering the feedback loop (frequency $\omega=4 \mathrm{~Hz}$ ). Open circles indicate that the signal $\phi-\phi_{0}$ has been accepted as periodic (the map $M_{1}$ is evaluated from the forcing interval before each open circle); the filled circles correspond to accepted fixed points of $M_{1}$ (and thus, to points on the curve shown in Fig. 4). The panels (b) and (c) show how the parameters $p$ and $\tilde{\phi}_{0}$ have been varied by the iteration (29).

error of the experimental result.

In our ongoing work we focus on extensions to mechanical hybrid tests $[4,10]$ and towards direct two-parameter continuation of certain bifurcations (such as the fold encountered in Fig. 4). More ambitious extensions are continuations of strongly non-harmonic motions (near so-called homoclinic or heteroclinic connections) or torus bifurcations.

\section{References}

[1] D. Barton and S.G. Burrow. Numerical continuation in a physical experiment: investigation of a nonlinear energy harvester. In Proceedings of the ASME 2009 International Design Engineering Technical Conferences \& Computers and Information in Engineering Conference, San Diego, number DETC2009-87318 in DETC, 2009.

[2] P.W. Bates, K. Lu, and C. Zeng. Persistence of overflowing manifolds for semiflow. Comm. Pure Appl. Math., 52(8), 1999.

[3] P.W. Bates, K. Lu, and C. Zeng. Invariant foliations near normally hyperbolic invariant manifolds for semiflows. Trans. Amer. Math. Soc., 352(10):4641-4676, 2000.

[4] A. Blakeborough, M.S. Williams, A.P. Darby, and D.M. Williams. The development of real-time substructure testing. Philosophical Transactions of the Royal Society of London A, 359:1869$1891,2001$.

[5] A. Dhooge, W. Govaerts, and Y.A. Kuznetsov. MatCont: a Matlab package for numerical bifurcation analysis of ODEs. ACM Transactions on Mathematical Software, 29(2):141-164, 2003.

[6] E.J. Doedel. Lecture notes on numerical analysis of nonlinear equations. In B. Krauskopf, H.M. Osinga, and J. Galán-Vioque, editors, Numerical Continuation Methods for Dynamical Systems: Path following and boundary value problems, pages 1-49. Springer-Verlag, Dordrecht, 2007.
[7] K. Engelborghs, T. Luzyanina, and G. Samaey. DDE-BIFTOOL v.2.00: a Matlab package for bifurcation analysis of delay differential equations. Report TW 330, Katholieke Universiteit Leuven, 2001.

[8] V. Eyert. A comparative study on methods for convergence acceleration of iterative vector sequences. Journal of Computational Physics, 124(0059):271-285, 1996.

[9] N. Fenichel. Geometric singular perturbation theory for ordinary differential equations. Journal of Differential Equations, 31:53-98, 1979.

[10] A. Gonzalez-Buelga, D.J. Wagg, and S.A. Neild. Parametric variation of a coupled pendulum-oscillator system using realtime dynamic substructuring. Structural Control and Health Monitoring, 14(7):991-1012, 2007.

[11] John Guckenheimer and Philip Holmes. Nonlinear oscillations, dynamical systems, and bifurcations of vector fields, volume 42 of Applied Mathematical Sciences. Springer-Verlag, New York, 1990. Revised and corrected reprint of the 1983 original.

[12] M. Hirsch, C. Pugh, and M.Shub. Invariant Manifolds, volume 583 of Lecture Notes in Mathematics. Springer-Verlag, Berlin, 1977.

[13] I.G. Kevrekidis, C.W. Gear, and G. Hummer. Equation-free: The computer-aided analysis of complex multiscale systems. AIChE Journal, 50(11):1346-1355, 2004.

[14] Yuri A. Kuznetsov. Elements of applied bifurcation theory, volume 112 of Applied Mathematical Sciences. Springer-Verlag, New York, third edition, 2004.

[15] K. Lust, D. Roose, A. Spence, and A.R. Champneys. An adaptive Newton-Picard algorithm with subspace iteration for computing periodic solutions. SIAM J. on Sci. Comp., 19(4):1188-1209, 1998.

[16] K. Nam and A. Arapostathis. A sufficient condition for local controllability of nonlinear systems along closed orbits. IEEE Transactions on Automatic Control, 37(3):378-380, 1992.

[17] K. Pyragas. Continuous control of chaos by self-controlling feedback. Phys. Lett. A, 170:421-428, 1992.

[18] D. Roose and R. Szalai. Continuation and bifurcation analysis of delay differential equations. In B. Krauskopf, H.M. Osinga, and J. Galán-Vioque, editors, Numerical Continuation Methods for Dynamical Systems: Path following and boundary value problems, pages 51-75. Springer-Verlag, Dordrecht, 2007.

[19] J. Sieber, A. Gonzalez-Buelga, S.A. Neild, D.J. Wagg, and B. Krauskopf. Experimental continuation of periodic orbits through a fold. Phys. Rev. Lett., 100(244101), 2008.

[20] J. Sieber and B. Krauskopf. Control based bifurcation analysis for experiments. Nonlinear Dynamics, 51(3):365-377, 2008.

[21] W. Szemplinská, E. Tyrkiel, and A. Zubrzycki. The global bifurcations that lead to transient tumbling chaos in a parametrically driven pendulum. International Journal of Bifurcation and Chaos, 10(9):2161-2175, 2000. 